\title{
Comparison of $\beta$-protein/A4 deposits and Alz-50-stained cytoskeletal changes in the hypothalamus and adjoining areas of Alzheimer's disease patients: amorphic plaques and cytoskeletal changes occur independently
}

Received: 22 September 1997 / Revised, accepted: 9 January 1998

\begin{abstract}
Alzheimer's disease is characterized neuropathologically by senile plaques and cytoskeletal changes. It has been proposed that amorphic plaques would locally induce anterograde propagation of cytoskeletal changes in consecutive neurons followed by amorphic plaque deposition at their axonal terminals. The Alzheimer changes would spread in this way along neural pathways. To test the 'primary amyloid anatomical cascade hypothesis', Congo red staining, $\beta$-protein/A4 (A $\beta)$ antiserum and Alz-50, which recognizes cytoskeletal changes, were applied to the hypothalamus and adjoining brain areas of five Alzheimer's disease patients of 40-90 years of age and five age- and sex-matched controls. The results showed that (1) virtually all $A \beta$ plaques in the hypothalamus were of the Congo red-negative amorphic type; (2) amorphic plaques and Alz-50-stained cytokeletal changes were observed not only in all Alzheimer's disease patients but also in a non-demented, 90-year-old control subject; (3) the density of amorphic plaques in the hypothalamus was unrelated to the duration of the dementia; (4) the density of amorphic plaques was unrelated to that of Alz-50stained cytoskeletal changes; (5) double-labeling with anti-A $\beta$ and Alz-50 did not show an evident topical relationship between amorphic plaque deposition and the occurrence of cytoskeletal changes; and (6) the distribution of $\mathrm{A} \beta$ and Alz-50 staining in five brain areas, for which
\end{abstract}

J. A. P. van de Nes · R. Ravid - D. F. Swaab

Netherlands Institute for Brain Research,

Amsterdam, The Netherlands

J. A. P. van de Nes

Department of Psychiatry, Valerius Clinic, Free University,

Amsterdam, The Netherlands

W. Kamphorst

Department of Neuropathology, Free University,

Amsterdam, The Netherlands

J. A. P. van de Nes (

Department of Pathology, University Hospital Nijmegen,

PO Box 9101, $6500 \mathrm{HB}$, Nijmegen, The Netherlands

Tel.: 31-24-3614314, Fax: 31-24-3540520 essential anatomical information is available, did not support the primary amyloid anatomical cascade hypothesis. Amorphic plaques and cytoskeletal changes rather occur independently.

Key words Alzheimer's disease $\cdot A l z-50 \cdot \beta$-protein . Cytoskeletal changes $\cdot$ Human hypothalamus

\section{Introduction}

$\beta$-protein [10] or A4-protein fibril (A $\beta$ ) deposition [19] in the neuropil has often been thought to be the primary and crucial event in the pathogenesis of Alzheimer's disease (AD). One of the hypotheses based on this idea is the 'primary amyloid anatomical cascade hypothesis' put forward by Hardy in 1992 [11]. This hypothesis states that amorphic plaques locally induce neurons to the development and anterograde propagation of cytoskeletal changes that accumulate in perikarya and dystrophic neurites. Subsequently, amorphic plaques would be formed at their axonal terminals. Amorphic plaques stain with $A \beta$ antibodies, but they do not have a congophilic core nor a corona of silver-stained dystrophic neurites as observed in neuritic and classic senile plaques (SPs) [25, 35]. Dystrophic neurites are short, thickened, curly or kinky processes termed 'neuropil threads' if they are localized in the neuropil outside SPs [7] - , which are visualized by means of sensitive silver stainings [7] or antibodies such as anti-tau Alz-50 [17, 41].

The hypothalamus in AD patients is a suitable brain structure to test the 'primary amyloid anatomical cascade hypothesis'. Firstly, the hypothalamus affected by $\mathrm{AD}$ was reported to contain many $A \beta$-positive Congo rednegative amorphic plaques that did not contain epitopes corresponding to regions of the $\beta$-amyloid precursor protein other than $A \beta$ [31]. Secondly, in the hypothalamus of AD patients the few neurofibrillary tangles (NFTs) and neuritic and classic SPs observed with conventional silver stainings $[13,14,26,28]$ are outnumbered by Alz-50stained cytoskeletal changes in perikarya and dystrophic 
neurites [37]. Thirdly, various hypothalamic and adjoining nuclei appeared to react in very different ways during the course of $\mathrm{AD}$, ranging from a total lack of staining with Alz-50 at one end to a high density of Alz-50-stained cytoskeletal changes at the other end of the spectrum [37].

The aim of the present study was to test the 'primary amyloid anatomical cascade hypothesis' by comparison of the density and distribution of amorphic plaques and Alz-50-stained cytoskeletal changes in various hypothalamic and adjoining nuclei in AD patients.

\section{Materials and methods}

Subjects and tissues

For this study the hypothalamus from five $\mathrm{AD}$ and five non-demented, age- and sex-matched control patients was used. The clinicopathological information on these patients is given in Table 1. The demented patients had been thoroughly examined in a clinical setting and were scored 6-7 for severity of dementia according to the Global Deterioration Scale (maximum score: 7) [23]. The controls had no primary neurological or psychiatric disorder. The clinical diagnosis of $\mathrm{AD}$ was confirmed neuropathologically on the basis of the presence of many SPs, NFTs and dystrophic neurites in Bodian- and Congo red-stained sections from the hippocampus and cortex (frontal area 10 or 11 ; temporal area 22 or the temporal pole; parietal area 7 or 40 ; occipital area 17 and 18). To exclude Parkinson changes the substantia nigra was also examined. Notably, the oldest control patient (no. 5) was clinically non-demented and neuropathologically not diagnosed as having AD since the presence of dystrophic neurites, NFTs and SPs were largely restricted to the hippocampus and temporal cortex and rarely seen in the parietal, frontal and occipital cortex.

The formalin-fixed, paraffin-embedded hypothalami was cut coronally into $6-\mu \mathrm{m}$-thick sections, which were stored at room temperature. Every 50th section was stained with thionin $(0.1 \%$ thionin in acetate, $\mathrm{pH} 4$ ) for general orientation. In addition, the suprachiasmatic nucleus (SCN) was visualized using anti-vasopressin [33] since this brain structure cannot be recognized in $6-\mu \mathrm{m}$-thin sections stained with thionin. Schematic drawings of the chiasmatic and tuberal region of the human hypothalamus in adults are given in Fig. 1.

\section{Congo red staining and antibodies used}

Congo red was used to detect apple-green birefringence to visualize $\beta$-amyloid cores (i.e., amyloid and classic SPs).
The following antibodies were used: 1G102 (Dako), directed against amino acid sequence $8-17$ of the A $\beta$ peptide (for information on the staining specificity see [2]); SP28 (generously obtained from Dr. B. Frangione, N.Y.) raised against amino acid sequence $1-28$ of the $A \beta$ peptide and recognizing amino acids 20-28 (for information of the staining specificity see [8]) - double staining with rabbit antiserum SP28 and mouse monoclonal Alz-50 was easier to perform than that with mouse monoclonal $A \beta$ antibody $1 \mathrm{G} 102-$; and monoclonal Alz-50 antibody (P-42, kindly donated by Abbott Laboratories, Chicago, Ill.), a mouse IgG clone isotype from the original IgM monoclonal produced by Wolozin et al. [41]. Alz-50 (IgG) had the same staining characteristics as the IgM clone [38, 39].

\section{Staining protocols}

Antibody 1G102 was used exclusively for single staining; SP28 was used for single staining and for double-labeling with Alz-50. Before staining with SP28 or 1G102, sections were pretreated with 98-100\% formic acid (Merck) for 20 min followed by five rinses in tap water for $10 \mathrm{~min}$ to improve $A \beta$ immunoreactivity [15]. Staining with SP28 $(1: 200)$ was visualized using the peroxidaseantiperoxidase (PAP) technique [32]. Staining with 1 G102 was visualized using a two-step peroxidase method using rabbit antimouse immunoglobulins (Dako, P260). Alz-50 single staining ( $1: 100)$ was performed as for $A \beta$ antibody $1 \mathrm{G} 102$ without formic acid pretreatment (see [37]). Single staining with SP28, 1G102 or

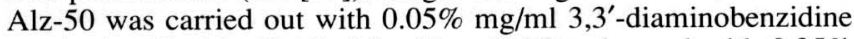
tetrahydrochloride (DAB; Merck) or DAB enhanced with $0.25 \%$ nickel ammonium sulphate (DAB-Ni), which gave a black precipitate. Selected sections were double-labeled with SP28 and Alz-50. Following formic acid pretreatment, SP28 (dilution $1: 200$ ) was used in the first sequence and $\mathrm{Alz}-50$ (1:75) in the second. $\mathrm{A} \beta$ staining was visualized with DAB only, while Alz-50 staining was achieved with DAB-Ni. In between the staining sequences, sections were incubated with methanol-hydrogen peroxide series according to Axelson and Van Leeuwen [3] to block residual horseradish peroxidase (HRP) activity of the first staining sequence. Following this step the yellow-brown staining of the SPs did not change to black, proving that no HRP activity was left.

\section{Results}

For specimens that were formalin-fixed for a period of up to 119 days (patient 7) or were obtained after a postmortem delay up to $24 \mathrm{~h}$ (patient 8 ), an intense $A \beta$ and $\mathrm{Alz}-50$ staining was seen in the hypothalamus of $\mathrm{AD}$ pa-
Table 1 Clinico-pathological information

AD: Alzheimer's disease; fix dur(d): fixation duration (in days); delay (h): post-mortem delay (in hours); $\mathrm{M}$ : male; F: female; demen $\operatorname{dur}(\mathrm{y})$ : dementia duration (in years); brain wght $(\mathrm{g})$ : brain weight (in grams)

\begin{tabular}{llllllll}
\hline & $\begin{array}{l}\text { Age } \\
(\mathrm{y})\end{array}$ & $\begin{array}{l}\text { Demen } \\
\text { dur(y) }\end{array}$ & $\begin{array}{l}\text { Sex } \\
(\mathrm{M} / \mathrm{F})\end{array}$ & $\begin{array}{l}\text { Brain } \\
\text { wght(g) }\end{array}$ & $\begin{array}{l}\text { Delay } \\
(\mathrm{h})\end{array}$ & $\begin{array}{l}\text { Fix } \\
\text { dur(d) }\end{array}$ & $\begin{array}{l}\text { Diagnosis, } \\
\text { causes of death }\end{array}$ \\
\hline Controls: & & & & & & & \\
1 & 37 & & $\mathrm{M}$ & 1510 & 48 & 46 & $\begin{array}{l}\text { Alcohol \& drugs abuse } \\
2\end{array}$ \\
43 & & $\mathrm{M}$ & 1260 & 23 & 53 & $\begin{array}{l}\text { Non-Hodgkin lymphoma } \\
\text { Mitral stenosis }\end{array}$ \\
3 & 57 & & $\mathrm{~F}$ & 1220 & 45 & 35 & Endocarditis lenta \\
4 & 72 & & $\mathrm{~F}$ & 1200 & 21 & 53 & Post-operative infections \\
5 & 90 & & $\mathrm{~F}$ & 1110 & 13 & 48 & \\
AD Patients: & & & & & & & \\
6 & 40 & $5-6$ & $\mathrm{M}$ & 1370 & 3 & 28 & AD, cachexia \\
7 & 45 & 10 & $\mathrm{M}$ & 1130 & 4 & 119 & Familial AD, cachexia \\
8 & 56 & $4-5$ & $\mathrm{~F}$ & 1180 & 24 & 48 & AD, bronchopneumonia \\
9 & 70 & 12 & $\mathrm{~F}$ & 780 & 14 & 34 & AD, epileptic insults \\
10 & 90 & 8 & $\mathrm{~F}$ & 1060 & 3 & 38 & AD, dehydration \\
\hline
\end{tabular}




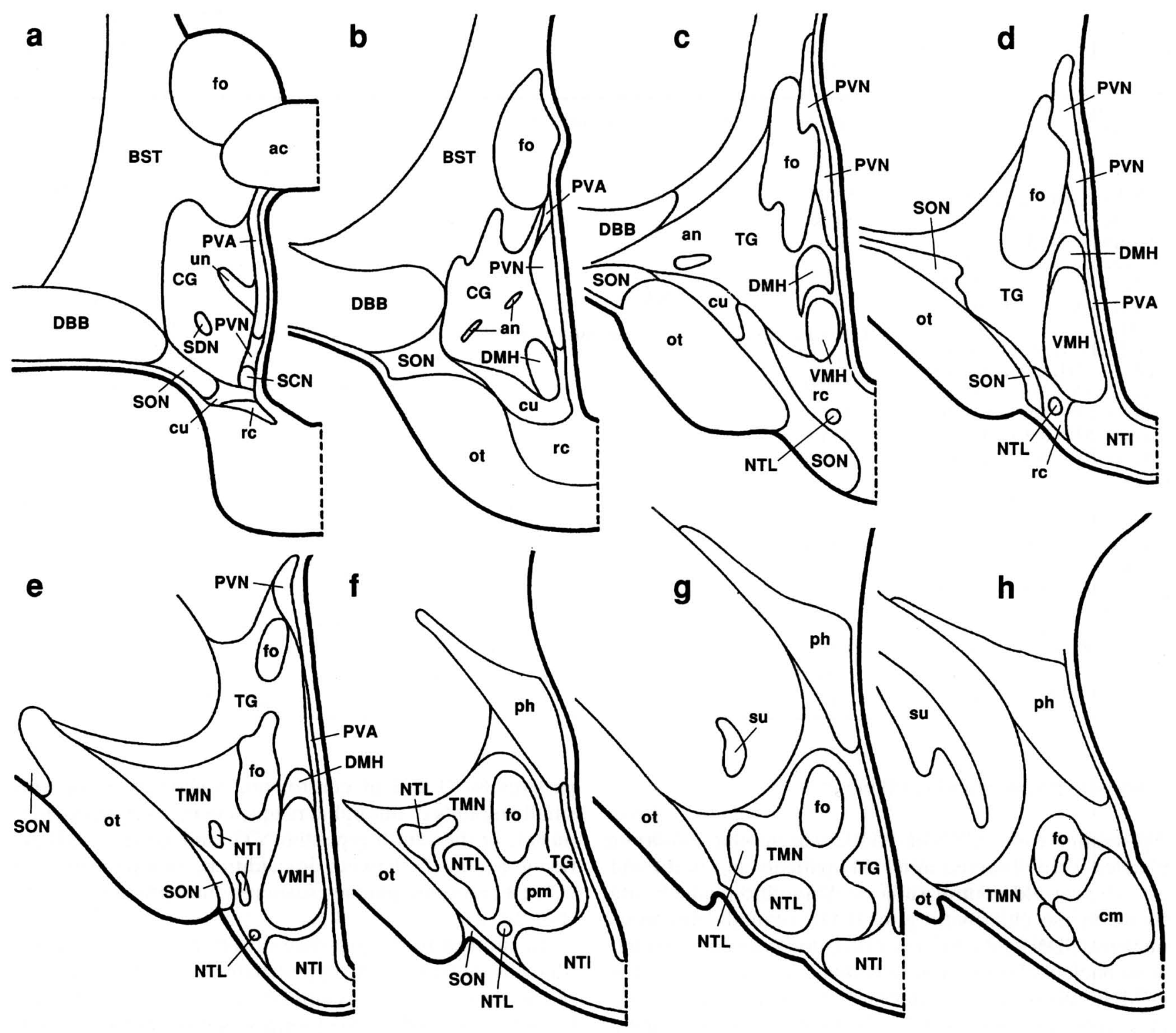

Fig. 1 Chiasmatic and tuberal region of the hypothalamus in the human adult (based upon [6], with kind permission from Elsevier Science). The diagrams show the main landmarks and nuclear grays that are encountered as coronal sections are traced anteroposteriorly. The distance between sections is $800 \mu \mathrm{m}$ ( $A C$ anterior commissure, $A N$ accessory neurosecretory nucleus, $C M$ corpus mamillare, $C U$ cuneate nucleus, $D B B$ nucleus of the diagonal band (of Broca), $D M H$ dorsomedial nucleus, $F M$ fasciculus mamillothalamica, $F O$ fornix, $C G$ chiasmatic gray, $T G$ tuberal gray, NTI infundibular nucleus, $S D N$ sexually dimorphic nucleus, $O C$ optic chiasm, $O T$ optic tract, $P V A$ periventricular nucleus, $P H$ posterior hypothalamic nucleus, $P M$ postero-medial nucleus, $P V N$ paraventricular nucleus, $R E$ retrochiasmatic nucleus, $S C N$ suprachiasmatic nucleus, $S O N$ supraoptic nucleus, $B S T$ nucleus of the stria terminalis, $S U$ subthalamic nucleus, $T H$ thalamus, $N T L$ lateral tuberal nucleus, TMN tuberomamillary nucleus, $U N$ uncinate nucleus, $V M H$ ventromedial nucleus)

tients and controls. Controls 1 and 3 with longer postmortem delays, 48 and $45 \mathrm{~h}$, respectively, did not show $\mathrm{A} \beta$ immunoreactivity. The negative $A \beta$ staining in these subjects was not due to the effect of postmortem decom- position since we obtained a good $A \beta$ staining in the hypothalamus of a 61-year-old AD patient with a postmortem delay of $84 \mathrm{~h}$.

\section{Morphology of the $A \beta$ staining}

Three types of $A \beta$ deposits were distinguished. In the first, reactive vessels were found only in the nucleus accumbens (ACC) and the central nucleus of the bed nucleus of the stria terminalis (BSTc) of AD patient 7, and in the tubero-mamillary nucleus (TMN) of AD patient 9. These $A \beta$ deposits showed a apple-green birefringence with Congo red, representing congophilic angiopathy. In the second, congophilic SP staining was so rare that they could not be scored, but the brain regions containing such SPs are indicated with an asterisk in Table 2. In the third type, most of the $A \beta$ immunoreactivity was found in $A \beta$ positive, Congo red-negative amorphic plaques. The following data concern this type of plaque. 
Table 2 Alzheimer's disease staining pattern of $\mathrm{A} \beta$ deposits

ACC nucleus accumbens, BST bed nucleus of the stria terminalis, BSTc central sector of the bed nucleus, BST1 lateral sector of the bed nucleus,

BSTm medial sector of the bed nucleus, CG chiasmatic gray, CI Calleja islands (in the nucleus accumbens), DBB diagonal band of Broca, DMH dorsomedial hypothalamic nucleus, NBM nucleus basalis of Meynert, NTI tubero-infundibular nucleus, NTL lateral tuberal nucleus, PVN paraventricular nucleus, SCN suprachiasmatic nucleus, SDS sexually dimorphic nucleus, SON supraoptic nucleus, TG tuberal gray, TMN tuberomammillary nucleus, VMH ventromedial hypothalamic nucleus

*: Congophilic cores were present but their amount was too low to score.

NA: not available; -: not or negligible staining; \pm : few;

+ : small amount; ++ : considerable amount; +++: large amount

\begin{tabular}{|c|c|c|c|c|c|c|c|c|c|c|}
\hline & \multicolumn{5}{|c|}{ Controls } & \multicolumn{5}{|c|}{ Alzheimer's disease patients } \\
\hline & 1 & 2 & 3 & 4 & 5 & 6 & 7 & 8 & 9 & 10 \\
\hline \multicolumn{11}{|c|}{ Chiasmatic region of the hypothalamus } \\
\hline SON & - & - & - & - & - & - & - & - & - & - \\
\hline $\mathrm{CG}$ & - & - & - & - & $++^{*}$ & $+^{*}$ & + & ++ & ++ & $++^{*}$ \\
\hline SDN & - & - & - & - & + & - & \pm & \pm & \pm & \pm \\
\hline $\mathrm{SCN}$ & - & - & - & - & - & - & - & - & - & - \\
\hline PVN & - & - & - & - & - & - & - & - & - & - \\
\hline PVA & - & - & - & - & - & - & - & - & - & - \\
\hline $\mathrm{ME}$ & - & NA & - & - & - & - & - & - & NA & - \\
\hline \multicolumn{11}{|c|}{ Tuberal region of the hypothalamus } \\
\hline $\mathrm{DMH}$ & - & - & - & - & \pm & \pm & \pm & \pm & - & \pm \\
\hline VMH & - & - & - & - & + & $+*$ & ++ & ++ & + & ++ \\
\hline TG & - & - & - & - & ++ & $+^{*}$ & $+^{*}$ & +++ & + & + \\
\hline TMN & - & - & - & - & + & $+++*$ & ++ & + & \pm & + \\
\hline NTL & - & - & - & - & \pm & $+++*$ & ++ & \pm & \pm & + \\
\hline NTI & - & - & - & - & - & \pm & \pm & - & - & \pm \\
\hline \multicolumn{11}{|c|}{ Adjoining areas } \\
\hline DBM & - & - & - & - & + & + & + & + & - & \pm \\
\hline DBB & - & - & - & - & + & + & + & \pm & - & - \\
\hline BSTc & - & - & - & - & \pm & $++^{*}$ & ++ & $+*$ & \pm & \pm \\
\hline BSTl & - & - & - & - & $\pm^{*}$ & $+++^{*}$ & +++ & ++ & \pm & \pm \\
\hline BSTm & - & - & - & - & \pm & + & + & ++ & - & + \\
\hline $\mathrm{ACC}$ & - & - & - & - & + & +++ & +++ & $++*$ & ++ & ++ \\
\hline CI & - & - & - & - & + & ++ & ++ & + & + & + \\
\hline
\end{tabular}

Amorphic plaque staining (Table 2)

A $\beta$ staining using SP28 or 1 G102 was similar. Amorphic plaques were observed in all AD patients and in the oldest, 90-year-old control subject. Amorphic plaques were found in the chiasmatic gray (CG), sexually dimorphic nucleus (SDN), tuberal gray (TG), dorsomedial hypothalamic nucleus (DMH), ventromedial hypothalamic nucleus (VMH), tubero-infundibular nucleus (NTI), TMN and nucleus tuberalis lateralis (NTL) in the hypothalamus, and in all subnuclei of the bed nucleus of the stria terminalis (BST), the nucleus basalis of Meynert (NBM) and diagonal band of Broca (DBB) in the basal forebrain, the nucleus accumbens (ACC) and islands of Calleja (CI) in the ACC. The supraoptic nucleus (SON), SCN, paraventricular nucleus (PVN), periventricular area (PVA) and median eminence (ME) were devoid of $A \beta$ staining. The same held true for white matter, i.e., the anterior commissure, internal capsule and fornix.

$A \beta$ staining could be subdivided into four different types. In the first, in the chiasmatic region (CG, SDN), tuberal region (TG, DMH, VMH, NTI, TMN) and the lateral (BSTl) and medial subnucleus of the BST (BSTm), most amorphic plaques were well demarcated and intensively stained in the younger AD patients (nos. 6 and 7 aged 40 and 45 years, respectively). In the second type, the same areas often had vague borders and a fine granular or dust-like aspect in the three older AD patients (nos. $8-10$ ) and in the non-demented, 90-year-old control (no. 5 ). In the third type of $A \beta$ staining, the NTL and BSTc of patients $8-10$ and of control no. 5 showed no or only small amounts of amorphic plaques in the periphery of the nuclei. In the fourth type, the NTL and BSTc of AD patients 6 and 7 showed, in addition, many intensively stained amorphic plaques scattered throughout the nuclei (Fig. 2c, d).

In contrast to the presence of different types of amorphic plaque types in the hypothalamus and BST, amorphic plaque morphology was similar in the ventral striatum or basal forebrain of all AD patients, and of control no. 5. In addition, the ACC and CI showed considerable amounts of patchy or ill-bordered globular amorphic plaques. The ACC-CI region was, therefore, easily distinguishable (Fig. 3d). The NBM and DBB in the basal forebrain showed only a small (cases 5-7) or a negligible or very low amounts (cases 8-10) of amorphic plaques (Fig. 3d).

\section{Alz-50 immunoreactivity (Table 3 )}

Application of Alz-50 revealed the presence of cytoskeletal changes, i.e., diffusely stained perikarya and dystrophic neurites. This type of Alz-50 staining was observed in all AD patients and in the 90-year-old non-demented control. Classic NFT-shaped structures were rarely observed. Recognition of these structures was obscured by the intense diffuse cell body staining and because of their globoid shape. The density of Alz-50stained cytoskeletal changes seen using the double-labeling method was not different from that using Alz-50 single 
Table 3 Alz-50 staining of dystrophic neurites (D) and perikarya $(\mathrm{P})$
The order of the letters D or P is related to the relative density of the Alz-50 positive dystrophic neurites of perikarya, respectively. The first letter presents the highest relative density.

For abbreviations see Table 2 . NA: not available; -: not or negligible staining; \pm : few; + : small amount; ++ : considerable amount; +++: large amount

\begin{tabular}{|c|c|c|c|c|c|c|c|c|c|c|}
\hline & \multicolumn{5}{|c|}{ Controls } & \multicolumn{5}{|c|}{ Alzheimer's disease patients } \\
\hline & 1 & 2 & 3 & 4 & 5 & 6 & 7 & 8 & 9 & 10 \\
\hline \multicolumn{11}{|c|}{ Chiasmatic region of the hypothalamus } \\
\hline SON & - & - & - & - & - & - & - & - & - & - \\
\hline $\mathrm{CG}$ & - & - & - & - & $\pm \mathrm{D}, \mathrm{P}$ & $+\mathrm{D}, \mathrm{P}$ & $\pm \mathrm{D}, \mathrm{P}$ & $\pm \mathrm{P}$ & $++\mathrm{D}, \mathrm{P}$ & $\pm \mathrm{D}, \mathrm{P}$ \\
\hline SDN & - & - & - & - & - & $+\mathrm{D}, \mathrm{P}$ & $\pm \mathrm{D}, \mathrm{P}$ & $\pm \mathrm{D}, \mathrm{P}$ & $++\mathrm{D}, \mathrm{P}$ & $+\mathrm{D}, \mathrm{P}$ \\
\hline $\mathrm{SCN}$ & - & - & - & - & - & $+\mathrm{P}, \mathrm{D}$ & $\pm \mathrm{P}$ & $\pm \mathrm{P}$ & $+\mathrm{D}, \mathrm{P}$ & $+\mathrm{P}$ \\
\hline PVN & - & - & - & - & - & - & - & - & $+\mathrm{P}, \mathrm{D}$ & - \\
\hline PVA & - & - & - & - & - & - & - & - & $+\mathrm{P}$ & - \\
\hline $\mathrm{ME}$ & - & NA & - & - & - & - & - & - & NA & - \\
\hline \multicolumn{11}{|c|}{ Tuberal region of the hypothalamus } \\
\hline DMH & - & - & - & - & $\pm \mathrm{D}, \mathrm{P}$ & $+\mathrm{D}, \mathrm{P}$ & $\pm \mathrm{D}, \mathrm{P}$ & $\pm \mathrm{D}, \mathrm{P}$ & $+\mathrm{D}, \mathrm{P}$ & $+\mathrm{D}, \mathrm{P}$ \\
\hline VMH & - & - & - & - & $\pm \mathrm{D}, \mathrm{P}$ & $+\mathrm{D}, \mathrm{P}$ & $\pm \mathrm{D}, \mathrm{P}$ & $++\mathrm{D}, \mathrm{P}$ & $+\mathrm{D}, \mathrm{P}$ & $\pm \mathrm{D}, \mathrm{P}$ \\
\hline TG & - & - & - & - & $\pm \mathrm{D}, \mathrm{P}$ & $+\mathrm{D}, \mathrm{P}$ & $\pm \mathrm{D}, \mathrm{P}$ & $\pm P$ & $++\mathrm{D}, \mathrm{P}$ & $+\mathrm{D}, \mathrm{P}$ \\
\hline TMN & - & - & - & - & $\pm \mathrm{D}, \mathrm{P}$ & $+++\mathrm{D}, \mathrm{P}$ & $+\mathrm{D}, \mathrm{P}$ & $++\mathrm{D}, \mathrm{P}$ & $++\mathrm{D}, \mathrm{P}$ & $+\mathrm{D}, \mathrm{P}$ \\
\hline NTL & - & - & - & - & $+\mathrm{D}, \mathrm{P}$ & $\pm \mathrm{D}, \mathrm{P}$ & $+\mathrm{D}, \mathrm{P}$ & $+++\mathrm{D}, \mathrm{P}$ & $+++\mathrm{D}, \mathrm{P}$ & $+++\mathrm{D}, \mathrm{P}$ \\
\hline NTI & - & - & - & - & - & - & - & - & - & - \\
\hline \multicolumn{11}{|c|}{ Adjoining areas } \\
\hline NBM & - & - & - & - & $\pm \mathrm{D}, \mathrm{P}$ & $++\mathrm{D}, \mathrm{P}$ & $++\mathrm{D}, \mathrm{P}$ & $++\mathrm{D}, \mathrm{P}$ & $++\mathrm{D}, \mathrm{P}$ & $++\mathrm{D}, \mathrm{P}$ \\
\hline DBB & - & - & - & - & $\pm \mathrm{D}, \mathrm{P}$ & $++\mathrm{D}, \mathrm{P}$ & $+\mathrm{D}, \mathrm{P}$ & $+\mathrm{D}, \mathrm{P}$ & $++\mathrm{D}, \mathrm{P}$ & $++\mathrm{D}, \mathrm{P}$ \\
\hline BSTc & - & - & - & - & $\pm \mathrm{D}, \mathrm{P}$ & $+\mathrm{D}, \mathrm{P}$ & $\pm \mathrm{P}$ & $+\mathrm{D}, \mathrm{P}$ & $+\mathrm{D}, \mathrm{P}$ & $+\mathrm{D}, \mathrm{P}$ \\
\hline BSTl & - & - & - & - & $\pm \mathrm{D}$ & $\pm \mathrm{D}, \mathrm{P}$ & - & $\pm \mathrm{D}, \mathrm{P}$ & $\pm \mathrm{D}, \mathrm{P}$ & $+\mathrm{D}, \mathrm{P}$ \\
\hline BSTm & - & - & - & - & $\pm \mathrm{P}$ & $+\mathrm{D}, \mathrm{P}$ & $\pm \mathrm{P}$ & $+\mathrm{D}, \mathrm{P}$ & $\pm \mathrm{D}, \mathrm{P}$ & $+\mathrm{D}, \mathrm{P}$ \\
\hline $\mathrm{ACC}$ & - & - & - & - & $\pm \mathrm{P}, \mathrm{D}$ & $\pm \mathrm{P}, \mathrm{D}$ & $\pm \mathrm{P}, \mathrm{D}$ & $\pm \mathrm{D}, \mathrm{P}$ & $+\mathrm{D}, \mathrm{P}$ & $+\mathrm{D}, \mathrm{P}$ \\
\hline CI & - & - & - & - & $+\mathrm{P}, \mathrm{D}$ & $\pm \mathrm{P}, \mathrm{D}$ & $\pm \mathrm{P}$ & $+\mathrm{D}, \mathrm{P}$ & $\pm \mathrm{P}$ & $+\mathrm{D}, \mathrm{P}$ \\
\hline
\end{tabular}

staining. There was a large spectrum in the density of cytoskeletal changes per nucleus, ranging from none in the SON to many in the NTL of AD patients. The NTL of the younger AD patients (cases 6 and 7 ) showed, however, low amounts of Als-50-reactive cytoskeletal changes (Fig. 3 a), while the older ones (cases 8-10) showed many such changes (Fig. $3 \mathrm{~b}$ ). The amount of Als-50-stained cytoskeletal changes in the ventral striatum (ACC, CI) of AD patients 6 and 7 was only slightly less than that in AD patients 8-10. In other nuclei no difference was found in the density of Alz-50-stained cytoskeletal changes between the younger and older AD patients.

Staining with Alz-50 in controls gave a pattern of mainly patchy granular perikarya and beaded fibers in particular areas in the hypothalamus (e.g., PVA, PVN, VMH, NTI, ME and all BST nuclei), as reported previously [40]. This staining in the controls is due to cross-reaction with a subpopulation of somatostatinergic neurons [41]. Distinction between the Alz-50 staining in controls and the cytoskeletal changes in AD patients was not difficult.

Comparison of the distribution of $\mathrm{A} \beta$ (SP28) and Alz-50 staining (cf. Tables 2, 3)

Amorphic plaque density did not correlate to that of Alz50-stained cytoskeletal changes. The hypothalamus and BST of the 45-year-old AD patient (no. 7) showed the highest numbers of $\mathrm{A} \beta$ deposits among the $\mathrm{AD}$ patients, while this brain region revealed the lowest number of Alz- 50-stained cytoskeletal changes. Note, moreover, that AD patient 9 had amounts of $A \beta$ deposits that were as low as those observed in the hypothalamus of the non-demented control no. 5, while the density of Alz-50-stained cytoskeletal changes ranked among the higher ones.

Many brain areas showed both amorphic plaques and cytoskeletal changes. However, there were quite a few exceptions to this rule. The $\mathrm{SCN}$ remained entirely negative for $\mathrm{A} \beta$ immunoreactivity, while in all $\mathrm{AD}$ patients it contained some Alz-50-stained cytoskeletal changes (cf. Fig. $3 \mathrm{c}$ ). The PVN and PVA were devoid of amorphic plaques in all AD patients. On the other hand, the PVN and PVA of AD patient 9 showed some Alz-50-stained cell bodies and dystrophic neurites. The NTI showed a few amorphic plaques in $\mathrm{AD}$ patients 6,7 and 10, while Alz-50-immunoreactive cytoskeletal changes were never observed in that nucleus of any of the AD patients. The SON and ME did not show staining with anti-A $\beta$ or Alz-50.

The SDN, DMH, BSTI and BSTm tended to show both amorphic plaques and cytoskeletal changes in the $\mathrm{AD}$ patients, but there were some exceptions. The SDN of AD patient 6 did not show amorphic plaques, but showed $\mathrm{Alz}$ 50-reactive cell bodies and dystrophic neurites; the SDN of control no. 5 contained amorphic plaques but no $\mathrm{Alz}$ 50-stained cytoskeletal changes (cf. Fig. 3e, f). The DMH and BSTm of AD patient 9 contained some Alz-50stained cytoskeletal changes, while amorphic plaques were not observed. On the other hand, the BSTl of AD patient 7 showed no Alz-50-stained cytoskeletal changes but contained many amorphic plaques. 
Fig. 2 a Alzheimer patient 6, 40 years of age; SP28/Alz-50 staining. Anti-A $\beta$ (SP28) staining in the tuberal gray $(T G)$ shows a low number of mainly large, intensely stained amorphic plaques $(a p)$. The amounts of Alz-50-stained perikarya $(p)$ and dystrophic neurites $(d n)$ are low. There is no clear local relationship between the distribution of amorphic plaques and cytoskeletal changes. b Alzheimer patient 8,56 years of age; anti-A $\beta$ (1G102) staining. Staining of amorphic plaques in the tuberal gray $(T G)$ is characterized by large amounts of often faintly granular and small deposits. c Alzheimer patient 7, 45 years of age; SP28/Alz-50 staining. The central part of the bed nucleus of the stria terminalis $(B S T c)$ can be easily delineated from the lateral sector of the bed nucleus (indicated with an asterisk). Arrows indicate faint granular $\beta / A 4-$ reactive garland-like $A \beta$ deposits in the rim of the nucleus. Amorphic plaques in the BSTc of AD patients are often more spheroid and more intensively stained with anti-A $\beta$. Cytoskeletal changes are not observed. d Alzheimer patient 9, 70 years of age, anti-A $\beta$ (1G102) staining. Again, the central sector of the bed nucleus of the stria terminalis $(B S T c)$ is obviously distinguishable from the surroundings, as indicated by arrows. Only in the periphery of the nucleus were some $A \beta$ deposits present. The surrounding nuclei are: 1 the caudate nucleus, 2 the lateral, and 3 the medial part of the bed nucleus of the stria terminalis ( $C G$ chiasmatic gray, $I C$ internal capsule, $l v$ lateral ventricle)

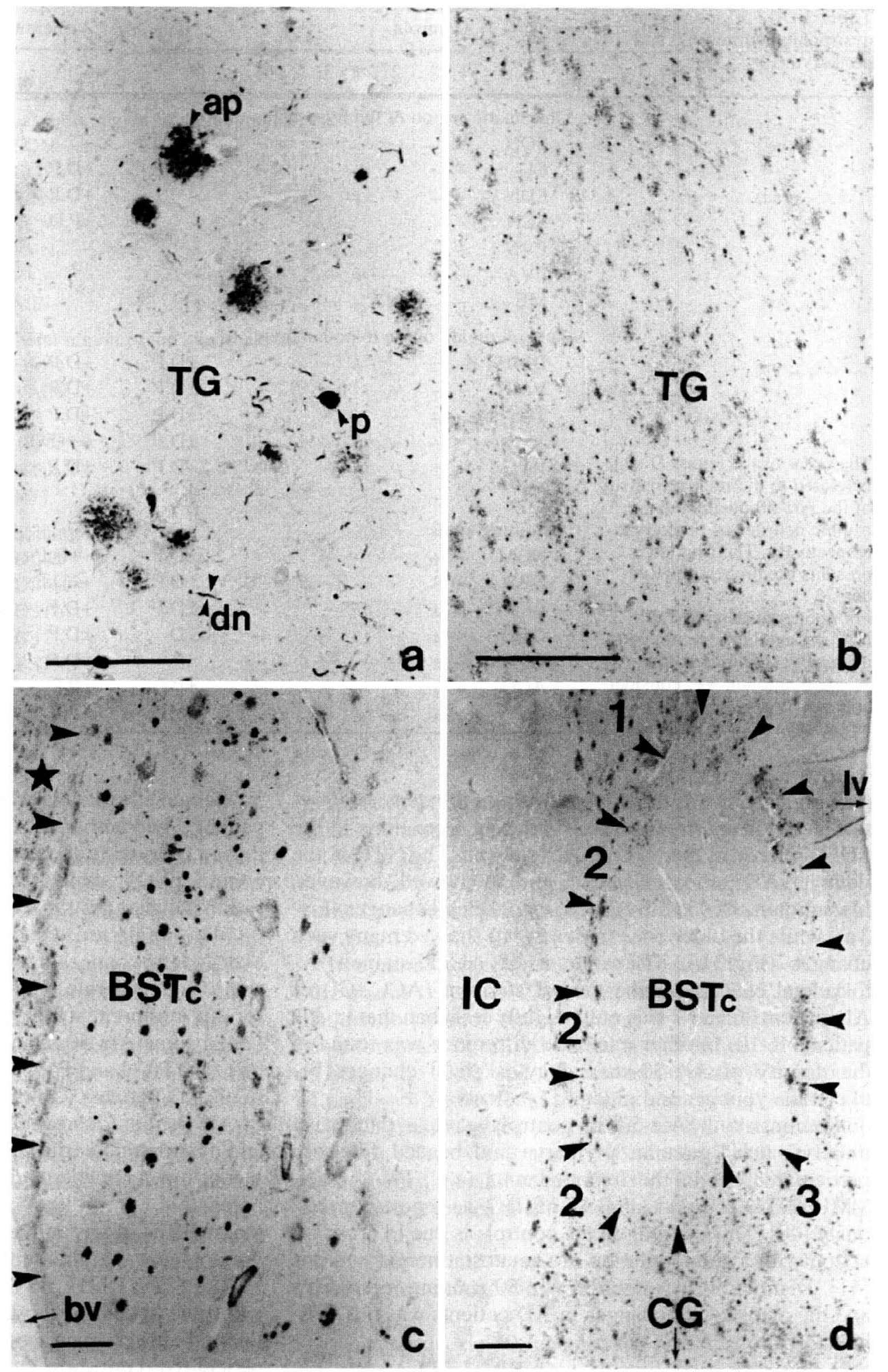

Double-labeling with anti-A $\beta$ and Alz-50 did not show any obvious local topical relationship between amorphic plaques and cytoskeletal changes.

\section{Discussion}

The hypothalamus and adjoining areas of AD patients have been reported to be affected merely by minute amounts of classic and amyloid SPs stained by conventional thioflavin S [26, 28] and Congo red staining [30]. 


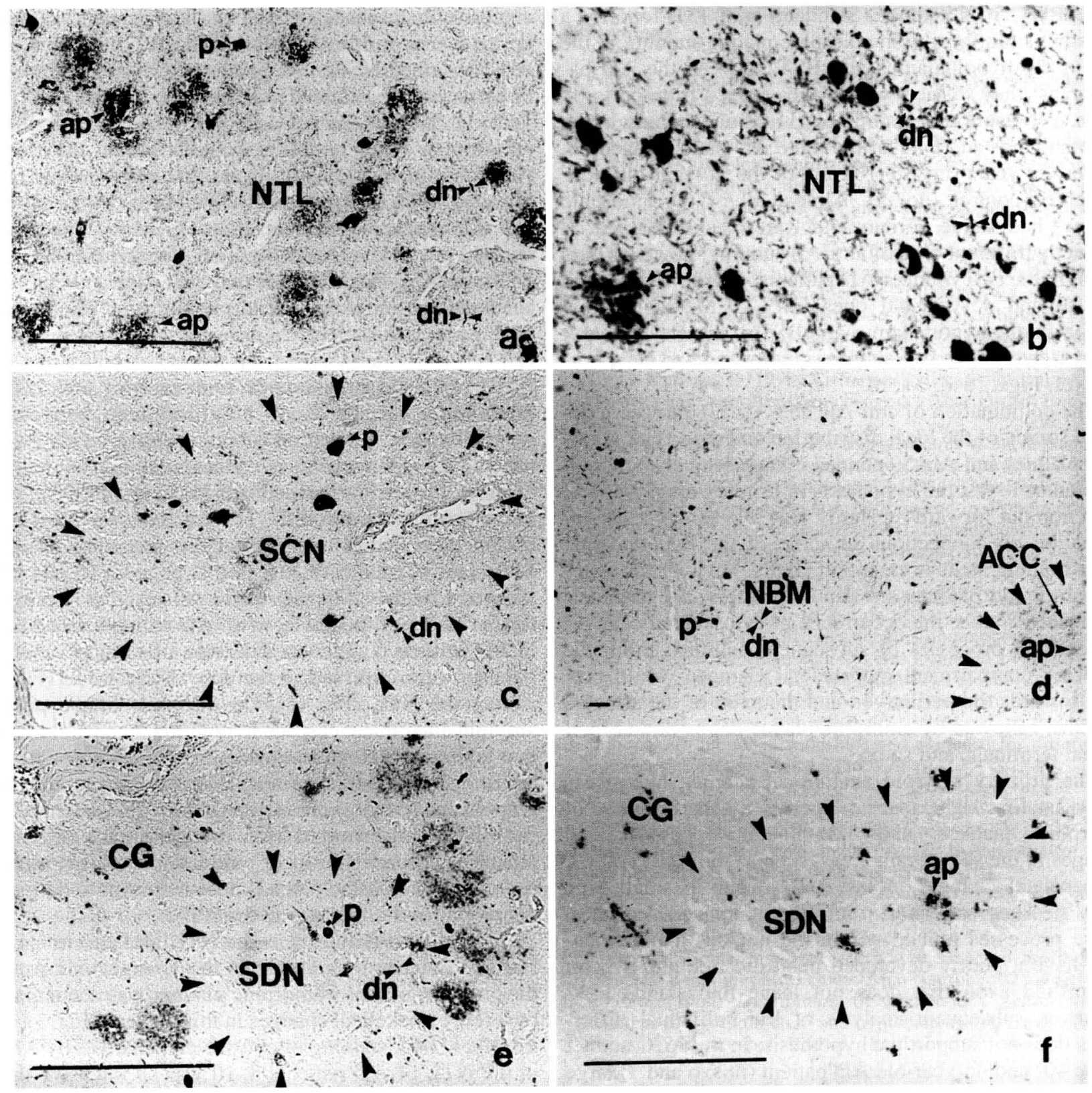

Fig. 3 a Alzheimer patient 6, 40 years of age; SP28/Alz-50 staining. The nucleus tuberalis lateralis $(N T L)$ of this young patient shows many amorphic plaques (ap) and few Alz-50-stained cytoskeletal changes. Most of the amorphic plaques are not intimately related to the few Alz-50-positive cell bodies or dystrophic neurites. b Alzheimer patient 10, 90 years of age; SP28/Alz-50 staining. The nucleus tuberalis lateralis (NTL) of this old patient stains heavily with Alz50 -stained cytoskeletal changes, but the amount of amorphic plaques (ap) is very low. These findings contrast those observed in a. c Alzheimer patient 9,70 years of age; SP28/Alz-50 staining. The suprachiasmatic nucleus ( $S C N)$ is indicated by arrows. It contains some perikarya $(p)$ and dystrophic neurites $(d n)$. However, A $\beta$-reac-

tive deposits are not observed. d Alzheimer patient 9, 70 years of age; SP 28/Alz-50 staining. The nucleus accumbens $(A C C)$ shows a high density of amorphic plaques ( $a p)$. The adjoining nucleus basalis of Meynert (NBM) contains many cell bodies $(p)$ and dystrophic neurites $(d n)$, but amorphic plaques are not found. e Alzheimer patient 6 , 40 years of age, SP28/Alz-50 staining. The sexual dimorphic nucleus $(S D N)$ contains some perikarya $(p)$ and dystrophic neurites $(d n)$. Amorphic plaques (ap) are found in the surrounding chiasmatic gray, but not within the SDN itself. f Non-demented control no. 5, 90 years of age; $1 \mathrm{G} 102$ staining. The SDN, indicated by arrows, does contain amorphic plaques (ap), in contrast to that of Alzheimer patient 6 (cf. e)

Our data confirm the presence of large amounts of amorphic plaques in these brain areas of AD patients, as reported by Standaert et al. [31]. In addition, we agree with Standaert et al. [31] that considerable $A \beta$ staining in the hypothalamus is not restricted to AD dementia. Amorphic

plaque density in the hypothalamus of $\mathrm{AD}$ patient 9 who had suffered from $\mathrm{AD}$ for more than 12 years was similar to that in the 90-year-old non-demented control (no. 5). Our data also show, in addition, that anti- $\mathrm{A} \beta$ staining was not only entirely negative in the SON and PVN, but also 
in the PVA, SCN and ME. Standaert et al. [31] reported a remarkable uniformity in amorphic plaque staining in the rest of the hypothalamus and BST between the AD patients that they studied, whose ages ranged from 65 to 88 years. We also found a similar $A \beta$ immunostaining in the hypothalamus and BST of the three AD patients aged 56, 70 and 90 years (nos. 8-10) and of the 90-year-old nondemented control (no. 5). Almost all of the amorphic plaques had vague borders and were weakly stained. A distinctly different type of anti-A $\beta$ staining, however, was found in the two younger $\mathrm{AD}$ patients, i.e. the 40 -year-old (no. 6) and 45-year-old ones (no. 7). Most amorphic plaques in the hypothalamus and BST of these early-onset $\mathrm{AD}$ patients showed an intense staining and had clear borders (cf. Figs. 2 a-d; 3 a, b).

The combination of anti-A $\beta$ and Alz-50 allowed a detailed survey of the local distribution and density of amorphic plaques and Alz-50-stained cytoskeletal changes and enabled us to test the hypothesis of primary amyloid local induction put forward by Hardy [11]. For this purpose information on connections in some of the hypothalamic and adjoining areas is essential. Hardy's hypothesis states that amorphic plaques are deposited at axonal terminals and locally induce the occurrence of cytoskeletal changes in dendritic processes of subsequent neurons. From the dendritic compartment cytoskeletal changes would proceed towards the perikaryon and the axon of the affected neurons, followed by amorphic plaque deposition at their axonal terminals, and so on.

The primary amyloid anatomical cascade hypothesis seems, at first sight, to be supported by the presence of amorphic plaques and Alz-50-stained cytoskeletal changes in the same brain areas, such as the ACC and CI. Cytoskeletal changes in the ACC occur in small type IV-V neurons with short ramifying axons and dendrites. These processes remain within the nucleus [5]. CI neurons have a poorly developed dendritic tree and a short unramified axon that does not leave the islands [22]. However, subsequent analysis of the individual differences does not support the hypothesis. In the ACC and CI of the 40- and 45-year-old AD patient (nos. 6 and 7) large amounts of amorphic plaques were found with only a very few Alz-50-stained cytoskeletal changes. In addition, the ACC of older AD patients aged 56, 70 and 90 years (nos. 8-10) contained fewer amorphic plaques, while cytoskeletal changes were present in similar or even higher densities than in the two younger AD patients. Moreover, the amorphic plaque densities in the CI of the 90 -year-old control (no. 5) were similar to those in the 70- and 90year-old AD patients (nos. 9 and 10), whereas Alz-50stained cytoskeletal changes in the CI of the 90-year-old, non-demented control (no. 5) were even more pronounced than those of the 70-year-old AD patient.

The observations in the NBM also cannot be explained by the amyloid cascade hypothesis. Magnocellular cholinergic neurons in the NBM [12] have direct connections with the cerebral cortex and hippocampus in primates [21]. In $\mathrm{AD}$, a functional deficit of these projections is likely to be partly responsible for the severe memory and cognitive disturbances. Large cholinergic neurons undergo extensive atrophy in $\mathrm{AD}[1,24,29]$ and show many Alz-50-stained NFTs, cell bodies and dystrophic neurites in all seriously affected $\mathrm{AD}$ patients $([34,37,41]$, cf. Table 3). The amount of amorphic plaques is, however, very low or virtually absent in the NBM of AD patients. Amorphic plaque density in the AD patients was rather low and, in fact, in the same range of that in the 90-yearold non-demented subject (no. 5), while this control showed only a few Alz-50-stained cytoskeletal changes.

The BSTc and NTL are two other areas suitable for checking the amyloid cascade hypothesis. These nuclei have some anatomical characteristics in common. Both can be clearly delineated from the enveloping areas. The BSTc and NTL show a dense fiber staining with anti-somatostatin $_{1-12}[9,18,36,38,40]$. In addition, both contain major somatostatin neuron subpopulations, which are distributed throughout these nuclei and maintain an intensive connectivity within these nuclei $[4,9,18,20,36]$. Staining with anti-somatostatin ${ }_{1-12}$ revealed densely distributed beaded fiber pericellular basket-like terminations in the BSTc $[18,40]$ and NTL $[36,38]$. Both the NTL and BSTc showed Congo red-negative and $A \beta$-reactive deposits in the periphery of the nuclei in all AD patients. In addition, in AD patients 6 and 7 considerable amounts of amorphic plaques were observed in the core of the BSTc (Fig. 2c) and of the NTL (Fig. $3 \mathrm{a}$ ). On the basis of the primary amyloid anatomical cascade hypothesis, one would expect that both nuclei contained many Alz-50-reactive cell bodies and dystrophic neurites. However, while the NTL showed many cytoskeletal changes in AD patients 8-10, the BSTc rarely showed such changes in any of the AD patients. Therefore, local induction of cytoskeletal changes by amorphic plaques does not seem to be the explanation for the findings in the BSTc.

Individual differences in the NTL also fail to support Hardy's cascade hypothesis. In the younger AD patients the presence of many amorphic plaques was accompanied by a few cytoskeletal changes in this nucleus (Fig. 3 a). On the other hand, staining of cytoskeletal changes by Alz-50 in the NTL of AD patients 8-10 was so intensive that it could be easily seen with the naked eye, while there was only little amorphic plaque staining in this nucleus (Fig. 3 b). Since dystrophic neurites stained by Alz-50 were found to hardly cross the border of the NTL [16, 34, 37], the vast majority of Alz-50-stained affected neurons in this nucleus would, according to Hardy's cascade hypothesis, be locally induced only by amorphic plaques within the nucleus itself. The data from the NTL do not support this possibility and rather seem to show an inverse relationship between amorphic plaque deposition and the occurrence of cytoskeletal changes. It is also not possible to explain why the NTL of young AD patients aged 40 and 45 years showed only few Alz-50-stained cytoskeletal changes, while the amount of amorphic plaques was high. On the other hand, the NTL of the older AD patients aged 56-90 years revealed many cytoskeletal changes, while amorphic plaque density was low in this nucleus. The number of amorphic plaques in the NTL of these three 
AD patients was as low as in the 90 -year-old control. This is more of a problem since no relationship was apparent between Alz-50 or $A \beta$ staining and disease duration. The occurrence of Alz-50-stained cell bodies and dystrophic neurites does not seem, therefore, to be locally induced by $A \beta$ nor does it seem to propagate through the neurons affected by amorphic plaques.

In summary, the distribution of $\mathrm{A} \beta$-reactive deposits and Alz-50-stained cytoskeletal changes appeared to be highly variable for (i) the two stainings, (ii) the various brain regions, and (iii) the different $\mathrm{AD}$ patients. The presence of a large number of amorphic plaques does not necessarily go together with many Alz-50-stained cytoskeletal changes. Amorphic plaque density in the CG and TG was high in the AD patients aged 56, 70 and 90 years, but the density of Alz-50-stained cytoskeletal changes did not differ in these nuclei between the two younger and the three older AD patients. The ACC contained many amorphic plaques, but few cytoskeletal changes. The reverse was found in the NBM, which showed many cytoskeletal changes, while amorphic plaques were sparse. In the NTL, the density of Alz-50-stained cytoskeletal changes tended to be inversely related to that of amorphic plaques and showed a cross-over in the course of aging. The ratio of Alz-50 and $A \beta$ staining in the ACC-CI, NBM, NTL and BSTc did not support the primary amyloid anatomical cascade hypothesis. Also, the observation that in the NTL there was not any local relationship between amorphic plaques and cytoskeletal changes is not in favor of the hypothesis. Taken together, $A \beta$ deposition and the occurrence of Alz-50-stained cytoskeletal changes rather seem to be independent phenomena. An alternative explanation is that amorphic plaque deposition is a risk factor rather than the causal factor for $\mathrm{AD}$. The variable way in which the different hypothalamic and adjoining nuclei are affected by cytoskeletal changes or spared in $\mathrm{AD}$ and controls might be due to their connectivity or different innate life span.

Acknowledgements This study is part of the AMSTEL project and was supported by the Stimuleringsprogramma GezondheidsOnderzoek (SGO) of the Ministry of Science and Education, The Netherlands. Brain material was obtained from the Netherlands Brain Bank of the Netherlands Institute for Brain Research, Amsterdam (Coordinator: Dr. R. Ravid). Alz-50 was kindly donated by Abbott Laboratories, Chicago, USA. SP28 was generously obtained from Dr. B. Frangione, New York, USA. The authors also wish to thank Mr. B. Fisser and Ms. H. B. J. M. Ploegmakers for technical assistance and Mr. G. van der Meulen for photographical work. Ms. A. Mulders (Department of Pathology of the Academic Hospital Nijmegen) is thanked for secretarial assistance. Postmortem neuropathological examinations were performed by Drs. W. Kamphorst (Hospital of the Free University, Amsterdam) and D. Troost (Academic Medical Center, Amsterdam).

\section{References}

1. Allen SJ, Dawbarn D, Wilcock GK (1988) Morphometric immunochemical analysis of neurons in the nucleus basalis of Meynert in Alzheimer's disease. Brain Res 454:275-281

2. Allsop D, Landon M, Kidd M, Lowe JS, Reynolds GP, Gardner A (1986) Monoclonal antibodies raised against a subsequence of senile plaque core protein react with plaque cores, plaque periphery and cerebrovascular amyloid in Alzheimer's disease. Neurosci Lett $68: 252-256$

3. Axelson JF, Van Leeuwen FW (1990) Differential localization of estrogen receptors in various vasopressin synthesizing nuclei of the rat brain. J Neuroendocrinol 2:209-216

4. Bouras C, Magistretti PJ, Morrison JH, Constantidinis J (1987) An immunohistochemical study of prosomatostatin-derived peptides in the human brain. Neuroscience 22:781-800

5. Braak H, Braak E (1990) Alzheimer's disease: striatal amyloid deposits and neurofibrillary changes. J Neuropathol Exp Neurol $49: 215-224$

6. Braak H, Braak E (1992) The hypothalamus of the human adult (chiasmatic and tuberal region). Prog Brain Res 93:3-16

7. Braak H, Braak E, Grundke-Iqbal I (1986) Occurrence of neuropil threads in the senile human brain and in Alzheimer's disease: a third location of paired helical filaments outside of neurofibrillary tangles and neuritic plaques. Neurosci Lett 65: 351-355

8. Castano EN, Ghiso J, Prelli F, Gorevic PD, Migheli A, Frangione B (1986) In vitro formation of amyloid fibrils from the synthetic peptides of different lengths homologous to Alzheimer's disease. Biochem Biophys Res Commun 141 : 782-789

9. Gaspar P, Berger B, Lesur A, Borsotti JP, Febvret A (1987) Somatostatin 28 and neuropeptide $\mathrm{Y}$ innervation in the septal area and related cortical and subcortical structures of the human brain. Distribution, relationships and evidence for differential coexistence. Neuroscience 22:49-73

10. Glenner GG, Wong CW (1984) Alzheimer's disease and Down's syndrome: share of a unique cerebrovascular amyloid fibril protein. Biochem Biophys Res Commun 122:1131-1135

11. Hardy J (1992) An 'anatomical cascade hypothesis' for Alzheimer's disease. Trends Neurosci 15:200-201

12. Hedreen JC, Struble RG, Whitehouse PJ, Price DL (1984) Topography of the magnocellular basal forebrain system in human brain. J Neuropathol Exp Neurol 43:1-21

13. Hirano A, Zimmerman HM (1962) Alzheimer's neurofibrillary changes: a topographical study. Arch Neurol 7:227-242

14. Ishii T (1966) Distribution of Alzheimer's neurofibrillary changes in the brain stem and hypothalamus of senile dementia. Acta Neuropathol (Berl) 6:181-187

15. Kitamoto T, Ogomori K, Tateishi J, Prusiner SB (1987) Formic acid pretreatment enhances immunostaining of cerebral and systemic amyloid. Lab Invest $57: 230-236$

16. Kremer HPH, Swaab DF, Bots GTAM, Fisser B, Ravid R, Roos RAC (1991) The hypothalamic lateral tuberal nucleus in Alzheimer's disease. Ann Neurol 29:279-284

17. Lee VM-L, Balin BJ, Orvos L, Trojanowski JQ (1991) A68: a major subunit of paired helical filaments and derivatized forms of normal tau. Science $251: 675-678$

18. Lesur A, Gaspar P, Alvarez C, Berger B (1989) Chemoanatomic compartments in the human bed nucleus of the stria terminalis. Neuroscience 32:181-194

19. Masters CL, Simms G, Weinman NA, Multhaup G, McDonald $\mathrm{BL}$, Beyreuther K (1985) Amyloid plaque core protein in Alzheimer disease and Down syndrome. Proc Natl Acad Sci USA $82: 4245-4249$

20. Mengod G, Rigo M, Savasta M, Probst A, Palacios JM (1992) Regional distribution of neuropeptide somatostatin gene expression in the human brain. Synapse 12:62-74 
21. Mesulam M-M, Mufson EJ, Levey AI, Wainer BH (1983) Cholinergic innervation of cortex by the basal forebrain; cytochemistry and cortical connections of the septal area, diagonal band nuclei, nucleus basalis (substantia innominata) and hypothalamus in the rhesus monkey. J Comp Neurol 214:170197

22. Meyer G, Gonzalez-Hernandez T, Carillo-Padilla F, FerresTorres R (1989) Aggregations of granule cells in the basal forebrain (islands of Calleja): Golgi and cytoarchitectonic study in different mammals, including man. J Comp Neurol 284:405428

23. Reisberg B, Ferris SH, De Leon MJ (1982) The Global Deterioration Scale for assessment of primary degenerative dementia. Am J Psychiatry 139: 1136-1139

24. Rinne JO, Paljarvi L, Rinne UK (1987) Neuronal size and density in the nucleus basalis of Meynert in Alzheimer's disease. J Neurol Sci 79:67-76

25. Rozemuller JM, Eikelenboom P, Stam FC, Beyreuther K, Masters CL (1989) A4 protein in Alzheimer's disease; primary and secondary cellular events in extracellular amyloid deposition. $\mathrm{J}$ Neuropathol Exp Neurol 48:674-691

26. Rudelli RD, Ambler MW, Wisniewski HM (1984) Morphology and distribution of Alzheimer neuritic (senile) and amyloid plaques in striatum and diencephalon. Acta Neuropathol (Berl) 64:273-281

27. Saper CB, Chelimsky TC (1984) A cytoarchitectonic and histochemical study of nucleus basalis and associated cell groups in the normal human brain. Neuroscience 13:1023-1037

28. Saper CB, German DC (1987) Hypothalamic pathology in Alzheimer's disease. Neurosci Lett 74:364-370

29. Salehi A, Lucassen PJ, Pool CW, Gonatas NK, Ravid R, Swaab DF (1994) Decreased neuronal activity in the nucleus basalis of Meynert in Alzheimer's disease as suggested by the size of the Golgi apparatus. Neuroscience 59871-880

30. Simpson J, Yates CM, Watts AG, Fink G (1988) Congo red birefringent structures in the hypothalamus in senile dementia of the Alzheimer type. Neuropathol Appl Neurobiol 14:381393
31. Standaert DG, Lee VM-Y, Greenberg BD, Lowery DE, Trojanowski JQ (1991) Molecular features of hypothalamic plaques in Alzheimer's disease. Am J Pathol 139:681-691

32. Sternberger LA (1979) Immunocytochemistry, 2nd. Edn. Wiley, New York

33. Swaab DF, Fliers E, Partiman TS (1985) The suprachiasmatic nucleus of the human brain in relation to sex, age and senile dementia. Brain Res 342:37-44

34. Swaab DF, Grundke-Iqbal I, Iqbal K, Kremer HPH, Ravid R, Van de Nes JAP (1992) Tau and ubiquitin in the human hypothalamus in aging and Alzheimer's disease. Brain Res 590: 239-249

35. Tagliavini F, Giaccone G, Frangione B, Bugiani O (1988) Preamyloid deposits in the cerebral cortex of patients with Alzheimer's disease and nondemented individuals. Neurosci Lett 93: 191-196

36. Timmers HJLM, Swaab DF, Van de Nes JAP, Kremer HPH (1996) Somatostatin S-12 $_{12}$ immunoreactivity is decreased in the hypothalamic lateral tuberal nucleus of Huntington's disease patients. Brain Res 728:141-148

37. Van de Nes JAP, Kamphorst W, Ravid R, Swaab DF (1993) $\mathrm{Alz}-50$ immunoreactivity in the hypothalamus and adjoining areas of Alzheimer's disease patients. Brain 115:103-115

38. Van de Nes JAP, Sluiter AA, Pool CW, Kamphorst W, Ravid R, Swaab DF (1994) The monoclonal antibody Alz-50, used to reveal cytoskeletal changes in Alzheimer's disease, also reacts with a large subpopulation of somatostatin neurons in the normal hypothalamus and adjoining areas. Brain Res 655:97-109

39. Vincent IJ, Davies P (1990) Phosphorylation characteristics of the A68 protein in Alzheimer's disease. Brain Res 531:127135

40. Walter A, Mai JK, Lanta L, Gorcs T (1991) Differential distribution of immunohistochemical markers in the bed nucleus of the stria terminalis in the human brain. J Chem Neuroanat 4: 281-298

41. Wolozin BL, Pruchnicki A, Dickson DW, Davies P (1986) A neuronal antigen in the brains of Alzheimer patients. Science $232: 648-650$ 